\title{
Tailorable and Repeatable Normal Contact Stiffness via Micropatterned Interfaces
}

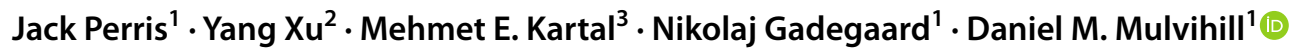

Received: 3 March 2021 / Accepted: 14 June 2021 / Published online: 17 July 2021

(c) The Author(s) 2021

\begin{abstract}
An approach to producing interfaces with tailored and repeatable normal contact stiffness using micropatterned surfaces is developed. A finite element model is first used to design square wave interfaces having a range of stiffnesses, and these are fabricated in polycarbonate via a microfabrication process. Results demonstrate that the contact stiffnesses of the fabricated interfaces are both tailorable and repeatable. The approach can be broadened to other materials and is useful for applications requiring specified interface stiffness. Finally, even with these deterministic interfaces, we show that low levels of roughness on the surface features are sufficient to produce a load-dependent contact stiffness at lower loads. Therefore, tailorability is mostly applicable above this limit where total contact stiffness converges to a load-independent value.
\end{abstract}

\section{Graphic Abstract}
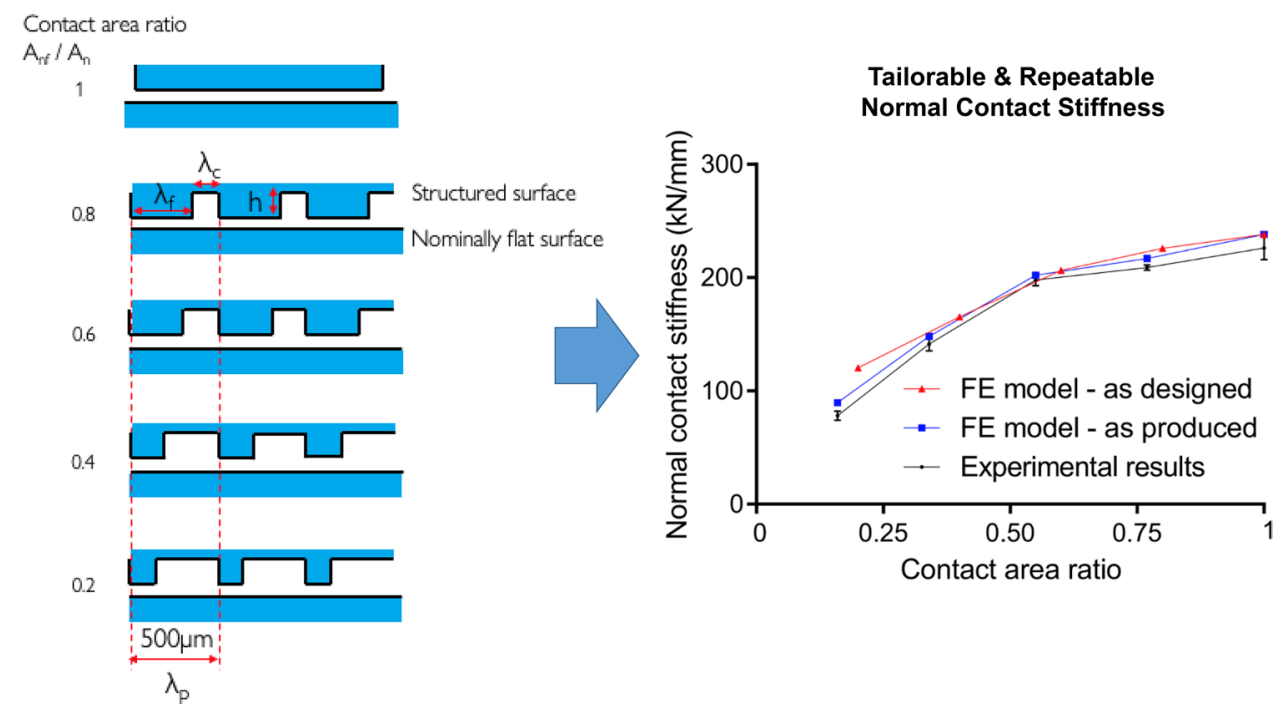

Keywords Contact stiffness $\cdot$ Structured surfaces $\cdot$ Micropattern $\cdot$ Microfabrication

\section{Introduction}

Yang Xu

Yang.Xu@hfut.edu.cn

$\triangle$ Daniel M. Mulvihill

daniel.mulvihill@glasgow.ac.uk

Extended author information available on the last page of the article

Normal contact stiffness $K$ is defined as the instantaneous rate of change of normal load $P$ with the relative approach $u$ of two surfaces in contact (i.e. $K=|\mathrm{d} P / \mathrm{d} u|$ ). Depending on how it is measured, it may be calculated based on deformation caused purely by the rough surface topography or it can be based on a combination of rough surface deformation 
and some amount of bulk material deformation occurring near the interface. The stiffness of a contact interface can be important in the design of mechanical systems depending on the application. It can affect the stability of a bone-implant interface [1], the vibration behaviour of joints [2], the electrical and thermal conductance of a contact interface [3, 4], the behaviour of robotic gripping systems [5, 6], and the performance and control of precision machinery [7]. Various advances have been made in the measurement of contact stiffness, and a number of approaches are available. Successful methods have been based on: ultrasound [8-10], laser interferometry [11], contact resonance [12] and digital image correlation [13-15]. Two main approaches have been taken to analytically model rough surface contact stiffness: asperity-based models [16, 17] built-up from the contact mechanics of single asperity interaction (i.e. Greenwood and Williamson's framework [18]) and the mean field approach based on overall surface statistics (i.e. Persson's approach [19]). Several advanced (and computationally intensive) computational approaches have also been deployed (e.g. finite element method [20], boundary element method [21] and Green's function molecular dynamics [22]). The models and experiments generally exhibit either a linear or power law contact stiffness variation with normal load depending on the contact details [22]. However, the accuracy of contact stiffness prediction for real engineering surfaces remains challenging due to the complexity of real contact scenarios and the simplifications inherent in the various modelling approaches. Repeatability is also a problem for real contact interfaces-e.g. a joint giving a different contact stiffness when reassembled. This makes it difficult to design interfaces where contact stiffness is a critical parameter. One example is the modelling of aeroengine vibrations. Very accurate finite element models exist to describe bulk component behaviour, but key interface parameters such as contact stiffness have to be empirically determined [23]. The random multiscale nature of the roughness present on most typical engineering surfaces is what makes the problem difficult. For example, Barber [24] showed that statistical fluctuations make a prediction of stiffness difficult if there is no separation between the scales of the macroscopic object and the longest wavelength of the roughness. In this paper we explore the possibility of using micropatterning to generate more deterministic surfaces with tailored and repeatable contact stiffness. The idea of using structured interfaces to tailor interface properties was recently explored for friction in Bin Jaber et al. [25] and for adhesive joints in Hamilton et al. [26]. The most relevant paper in relation to contact stiffness is that by Li et al. [27]. Li et al. explored the contact stiffness of an array of rigid cylindrical micropillars impinging a flat deformable surface. Using elastic flat-punch theory, they showed how interaction between impinging pillars leads to a contact stiffness dependent on, not just pillar spacing, but also on the number of contacting pillars (or equivalently, the apparent size of the overall nominal contact region). In this paper, we begin with a finite element approach to first design a set of micropatterned surfaces with varying instances of contact stiffness. We then use photolithography, etching and injection moulding steps to fabricate these surfaces (in polycarbonate-PC) and explore the extent to which the contact stiffnesses of the real as-produced surfaces replicate the design surfaces. Digital image correlation local to the contact interface is used to accurately measure displacements (and, hence, contact stiffness). We also explore some of the key factors effecting the realisation of an adjustable tailored contact stiffness: apparent contact area (or equivalently feature width in this case), feature geometric imperfections and the presence of nano-roughness on the feature tops of the micropatterned surfaces. The repeatability of the contact stiffness for different interface sample sets produced using the same mould tooling is also investigated.

\section{Design Interfaces}

A square wave pattern in contact with a flat surface of the same material (Fig. 1) was used for the investigationmainly because the square wave topography minimises some

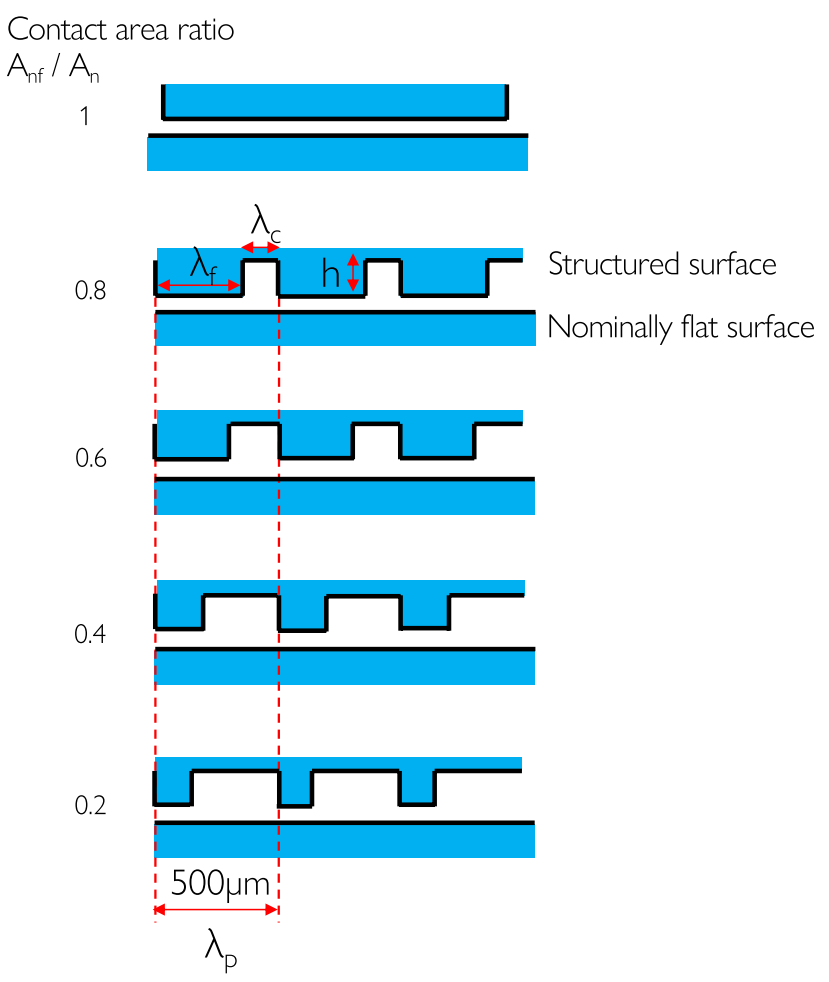

Fig. 1 Schematic representation of square wave instances at different design contact area ratios. Contact area ratio $A_{\mathrm{nf}} / A_{\mathrm{n}}=\lambda_{\mathrm{f}} / \lambda_{\mathrm{p}}$. Overall nominal contact area is $10 \times 10 \mathrm{~mm}$ 
of the complexity that can arise in fabrication and makes adjustment of surface feature parameters relatively straightforward. A thermoplastic polymer (polycarbonate) was used for both interface samples as highly accurate patterns can be injection moulded (see Sect. 3). The unstructured flat-on-flat case was also included as a benchmark. We first defined a series of square wave instances designed to produce a range of different design normal contact stiffnesses. This was done by adjusting the square wave feature width $\lambda_{\mathrm{f}}$ while holding the period $\left(\lambda_{\mathrm{p}}=500 \mu \mathrm{m}\right)$ and overall nominal contact size $\left(10 \times 10 \mathrm{~mm}^{2}\right)$ constant. The feature width was adjusted to give nominal contact area ratios of $0.2,0.4,0.6,0.8$ and 1 as shown in Fig. 1. Contact area ratio is defined here as the total nominal feature contact area $A_{\text {nf }}$ (i.e. the tops of the features) divided by the nominal area $A_{\mathrm{n}}$ (i.e. total enclosed planar area of $10 \times 10 \mathrm{~mm}^{2}$ ). Note $A_{\mathrm{nf}} / A_{\mathrm{n}}$ is equivalent to $\lambda_{\mathrm{f}} / \lambda_{\mathrm{p}}$. Feature height was held constant at $h=50 \mu \mathrm{m}$. Design dimensions are given in Table 1. A linear elastic finite element model (Fig. 2) was then used to determine the associated design contact stiffness for each case, and these are listed in Table 2. For calculation of the FE contact stiffness, node pairs on either side of the interface separated by the same distance $d=200 \mu \mathrm{m}$ as for the calculation of experimental contact stiffness (described later in Sect. 4

Table 1 Changes in key structured surface dimensions (with a bearing on contact stiffness) from design values to as-produced polycarbonate (PC) surfaces

\begin{tabular}{|c|c|c|c|c|c|c|c|c|c|c|}
\hline \multicolumn{4}{|c|}{ Feature depth $(h)$} & \multicolumn{4}{|c|}{ Feature width $\left(\lambda_{\mathrm{f}}\right)$} & \multicolumn{3}{|c|}{ Contact area ratio } \\
\hline $\begin{array}{l}\text { Design } \\
(\mu \mathrm{m})\end{array}$ & $\begin{array}{l}\text { Silicon } \\
(\mu \mathrm{m})\end{array}$ & $\begin{array}{l}\text { as-produced PC } \\
(\mu \mathrm{m})\end{array}$ & $\begin{array}{l}\% \\
\text { Increase }\end{array}$ & Design $(\mu \mathrm{m})$ & $\begin{array}{l}\text { Silicon } \\
(\mu \mathrm{m})\end{array}$ & $\begin{array}{l}\text { as-produced PC } \\
(\mu \mathrm{m})\end{array}$ & $\begin{array}{l}\% \\
\text { Decrease }\end{array}$ & Design & as-produced PC & $\begin{array}{l}\% \\
\text { Decrease }\end{array}$ \\
\hline 50 & $50.8 \pm 0.3$ & $52.3 \pm 0.7$ & 4.5 & 120 & $126.8 \pm 2.9$ & $80.6 \pm 1.9$ & 32.8 & 0.20 & 0.16 & 20 \\
\hline 50 & $52.5 \pm 0.3$ & $52.8 \pm 0.6$ & 5.7 & 225 & $225.3 \pm 2.6$ & $171.4 \pm 3.2$ & 23.8 & 0.40 & 0.34 & 15 \\
\hline 50 & $52.8 \pm 0.4$ & $53.9 \pm 0.7$ & 7.9 & 330 & $323.8 \pm 2.3$ & $275.4 \pm 3.8$ & 16.6 & 0.60 & 0.55 & 8.3 \\
\hline 50 & $49.7 \pm 0.4$ & $51.1 \pm 0.5$ & 2.2 & 440 & $439.9 \pm 2.6$ & $387.8 \pm 3.7$ & 11.9 & 0.80 & 0.77 & 3.8 \\
\hline
\end{tabular}

Table shows evolution of feature depth $h$, feature width $\lambda_{\mathrm{f}}$ and contact area ratio. For feature depth and feature width, the corresponding dimension for the intermediate silicon master is also indicated

Fig. 2 Finite element model (linear elastic) for determination of contact stiffnesses

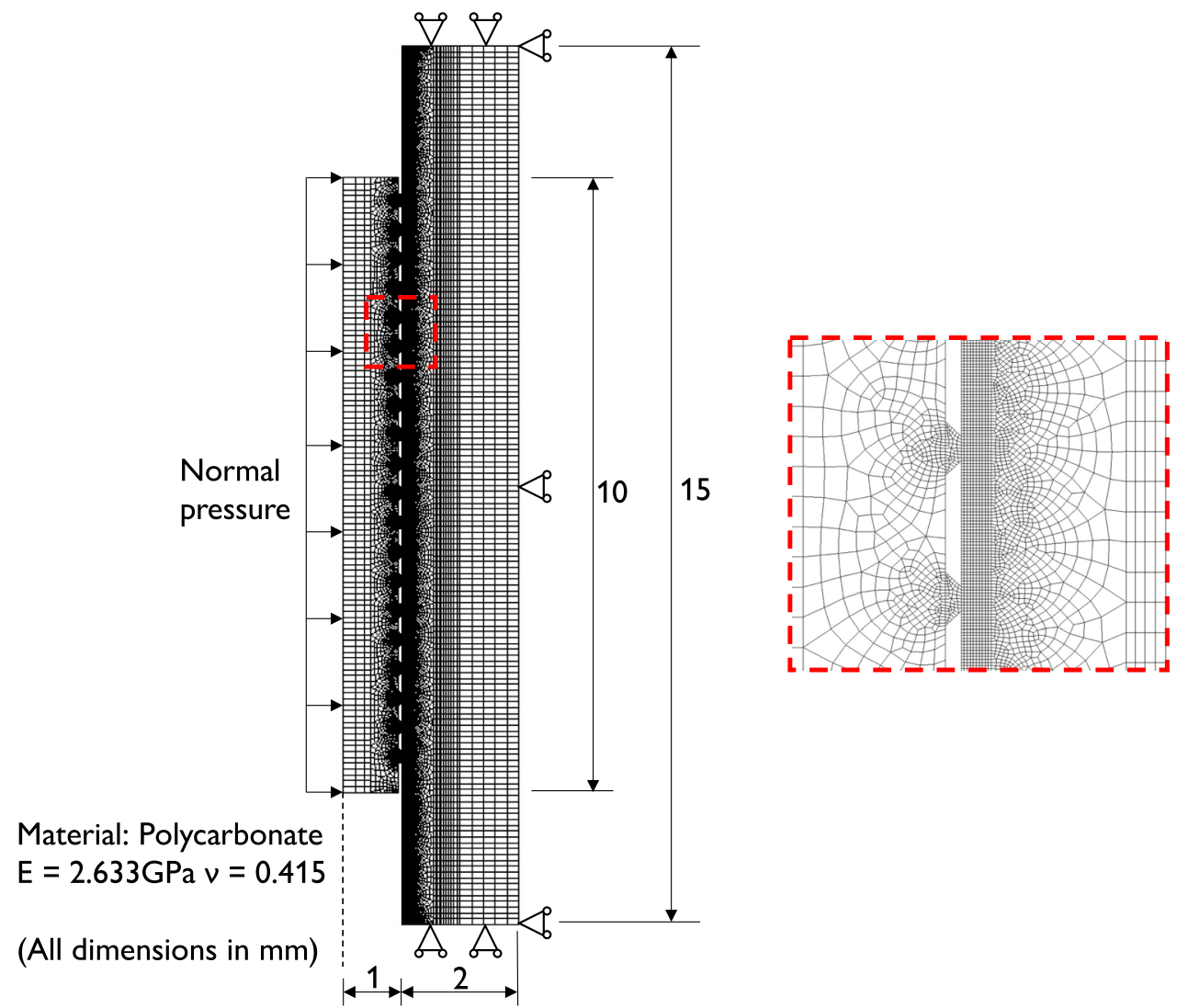


Table 2 Comparison of experimentally measured (total) normal contact stiffness (at saturation) with finite element determined contact stiffness for 'as-designed' and modified 'as-produced' cases

\begin{tabular}{|c|c|c|c|c|c|c|c|}
\hline \multicolumn{2}{|c|}{ FE—as designed } & \multicolumn{2}{|c|}{$\mathrm{FE}$ - as produced } & \multicolumn{2}{|c|}{ Experimental } & \multirow{2}{*}{$\begin{array}{l}\text { \% Drop from 'as- } \\
\text { designed FE' }\end{array}$} & \multirow{2}{*}{$\begin{array}{l}\% \text { Drop from } \\
\text { 'as-produced' } \\
\text { FE }\end{array}$} \\
\hline $\begin{array}{l}\text { Contact } \\
\text { area ratio }\end{array}$ & $\begin{array}{l}\text { Normal contact stiff- } \\
\text { ness, } K(\mathrm{kN} / \mathrm{mm})\end{array}$ & $\begin{array}{l}\text { Contact } \\
\text { area ratio }\end{array}$ & $\begin{array}{l}\text { Normal contact stiff- } \\
\text { ness, } K(\mathrm{kN} / \mathrm{mm})\end{array}$ & $\begin{array}{l}\text { Contact } \\
\text { area ratio }\end{array}$ & $\begin{array}{l}\text { Normal contact stiff- } \\
\text { ness, } K(\mathrm{kN} / \mathrm{mm})\end{array}$ & & \\
\hline 0.2 & 120.4 & 0.16 & 89.7 & 0.16 & $78.1 \pm 4.0$ & 35.1 & 12.9 \\
\hline 0.4 & 165.4 & 0.34 & 148.2 & 0.34 & $141.5 \pm 5.9$ & 14.5 & 4.5 \\
\hline 0.6 & 206.4 & 0.55 & 202.2 & 0.55 & $197.9 \pm 5.1$ & 4.1 & 2.1 \\
\hline 0.8 & 225.9 & 0.77 & 216.8 & 0.77 & $208.9 \pm 2.2$ & 7.6 & 3.6 \\
\hline 1 & 238.2 & 1 & 238.2 & 1 & $226.2 \pm 10.3$ & 5.1 & 5 \\
\hline
\end{tabular}

and Fig. 6) were used. This measure of contact stiffness, of course, includes a bulk material contribution. As shown (in Table 2), the various surface instances give contact stiffnesses ranging from 120.4 to $238.2 \mathrm{kN} / \mathrm{mm}$. The challenge is then to determine the extent to which it is possible to replicate these stiffness values repeatably in fabricated samples.

\section{Fabrication}

Both interface samples (each polycarbonate) were fabricated via injection moulding. This section outlines the steps involved. The square wave pattern was first produced on a silicon master. Standard photolithography was used to generate a grid pattern on a photoresist coating. Deep reactive ion etching (DRIE) was then used to etch the exposed regions in a highly directional process resulting in highly vertical sidewalls. The process is outlined in Fig. 3.

The silicon master is not suitable for use as a mould inlay. Therefore, the second step in the process involves transferring the pattern from the silicon master into a material with suitable thermal properties and durability for use as a mould inlay. This was done using an adapted nanofabrication protocol: Fig. 4 conveys the key stages. The silicon master is covered in an anti-stick layer (to enable separation of the wafer and stamp material) with a substance known as the working stamp material spun on top of the wafer (Fig. 4a). The wafer is then placed in a tool where the working stamp layer is imprinted onto a new transparent layer of polyethylene terephthalate
Fig. 3 Overview of silicon square wave microfabrication: development of a pattern in a photoresist coating via photolithography followed by etching of exposed regions to produce the square wave pattern in the silicon itself

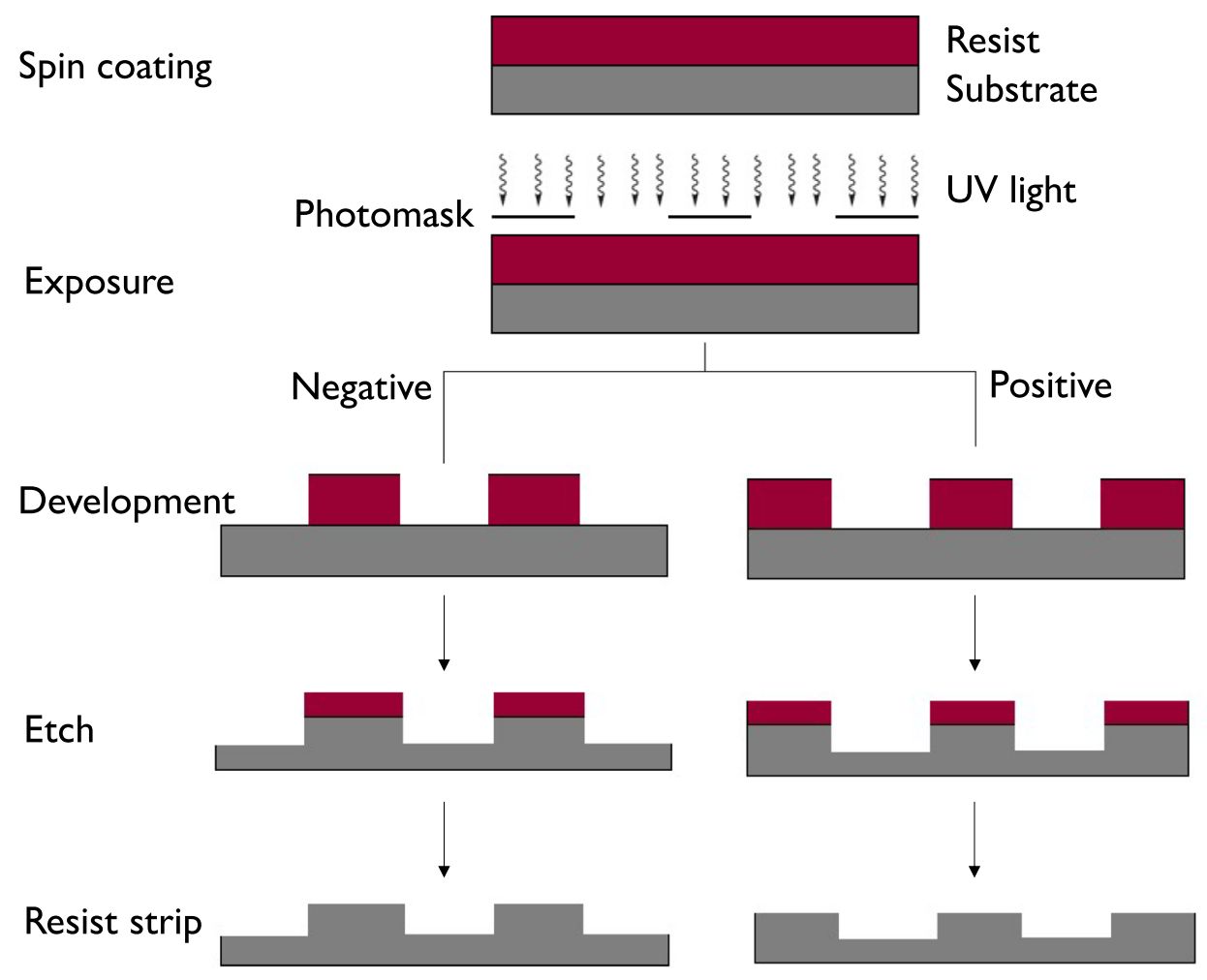


Fig. 4 Creation of mould inlay and injection moulding of polycarbonate samples: a spinning of a working stamp EVG foil material (purple) on top of the structured silicon master-an anti-stick layer (red) is spun initially to enable separation from the silicon substrate; $\mathbf{b}$ imprinting of the microfeatures from the silicon to the EVG foil followed by UV curing to solidify the mould insert; $\mathbf{c}$ imprinted micropatterned EVG foil; and $\mathbf{d}$ injection moulding of polycarbonate samples (blue) using the mould insert from $\mathbf{c}$ (Colour figure online)
Anti-stick layer

EVG foil

Polymer interface

(a)

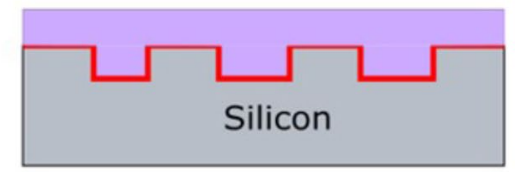

(b)

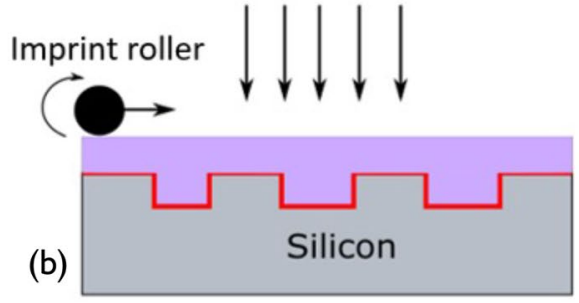

EVG foil is peeled from silicon substrate

Injection moulding of polymer structures using the EVG foil as the mould inlay

(c)

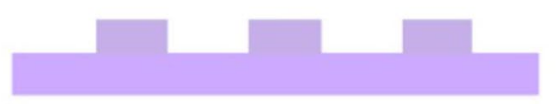

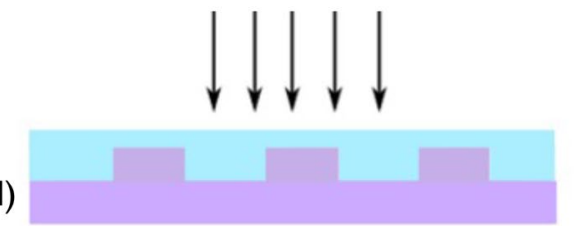

UV light
(PET) and subsequently UV-cured (Fig. 4b), producing the inverse pattern from the silicon wafer on the new PET sheet (Fig. 4c). The structured foil is then laser cut to the size required for the mould inlay.

The final step in the fabrication process uses injection moulding to obtain the microstructured pattern (Fig. 4d) in polycarbonate. The key parameters that characterise the moulding process are mould temperature, polymer injection temperature, injection velocity, pressure and the cooling time. Optimising the moulding conditions ensures sufficient filling of the microcavities to replicate the geometric fidelity from silicon master to polycarbonate. Injection moulding was performed using an Engel Victory 28 fully hydraulic injection moulding machine with a melt temperature of $270{ }^{\circ} \mathrm{C}$ and a tool temperature of $70{ }^{\circ} \mathrm{C}$. The PET foil supporting the pattern was laser cut to dimensions of $24.5 \mathrm{~mm} \times 24.5 \mathrm{~mm}$ and placed inside a tool hardened steel frame. The polycarbonate was dried for a minimum of $2 \mathrm{~h}$ at $110{ }^{\circ} \mathrm{C}$ in a vacuum oven prior to moulding. More extensive information on the moulding route used here is given in Hamilton et al. [28].

The samples were characterised during the fabrication process to allow analysis and comparison of the stages in the manufacturing process. The feature widths, heights and channel widths were all measured using optical profilometry (Contour GT, Bruker, US). The critical measurements were taken from the silicon masters, moulding inlays and polycarbonate replicas. Scans were taken at five locations across the respective samples. Multiple measurements of the critical dimensions were then taken from each scan of the samples.

\section{Experiments}

A micromechanical test machine ( $5 \mathrm{kN}$ Dual leadscrew, Deben, UK) was used to apply normal load to the samples via custom-designed self-aligning fixtures (Fig. 5). A nominally flat polycarbonate sample was glued to the flat platen on the left side of the arrangement, and the polycarbonate countersurface (patterned or flat) was glued to the platen on the right side attached to a ball and socket joint. The ball and socket joint facilitates self-alignment of the interface to ensure uniform contact. The fixtures were also designed so that the central (perpendicular) axis of the polymer samples is in line with the machine's load cell line-of-action. Tests were conducted with ambient conditions of temperature, pressure and humidity, and samples were cleaned via IPA and compressed air prior to testing. An initial load of $5 \mathrm{~N}$ is used to align the surfaces. The contact is then loaded up to $1500 \mathrm{~N}$ at a rate of $0.5 \mathrm{~mm} /$ min (i.e. quasistatic), and the load was recorded via the machine's load cell. Relative normal interface displacement was measured local to the interface using digital image correlation (DIC) by tracking the relative approach of target pairs close to the interface (separated initially by approximately $d=200 \mu \mathrm{m}$ ) as illustrated in Fig. 6. Five corresponding target pairs were used to facilitate calculation of an average value (only 3 are shown in Fig. 6). To capture the images for DIC, a side-on video of the interface region was captured using a digital camera (PLD732 2.2MP, Pixelink) and high-magnification adjustable lens system (Zoom 6000, Navitar, USA) positioned above 
Fig. 5 Experimental test rig for application of normal load: micromechanical test machine with custom-designed selfaligning fixtures (dashed red box) (Colour figure online)

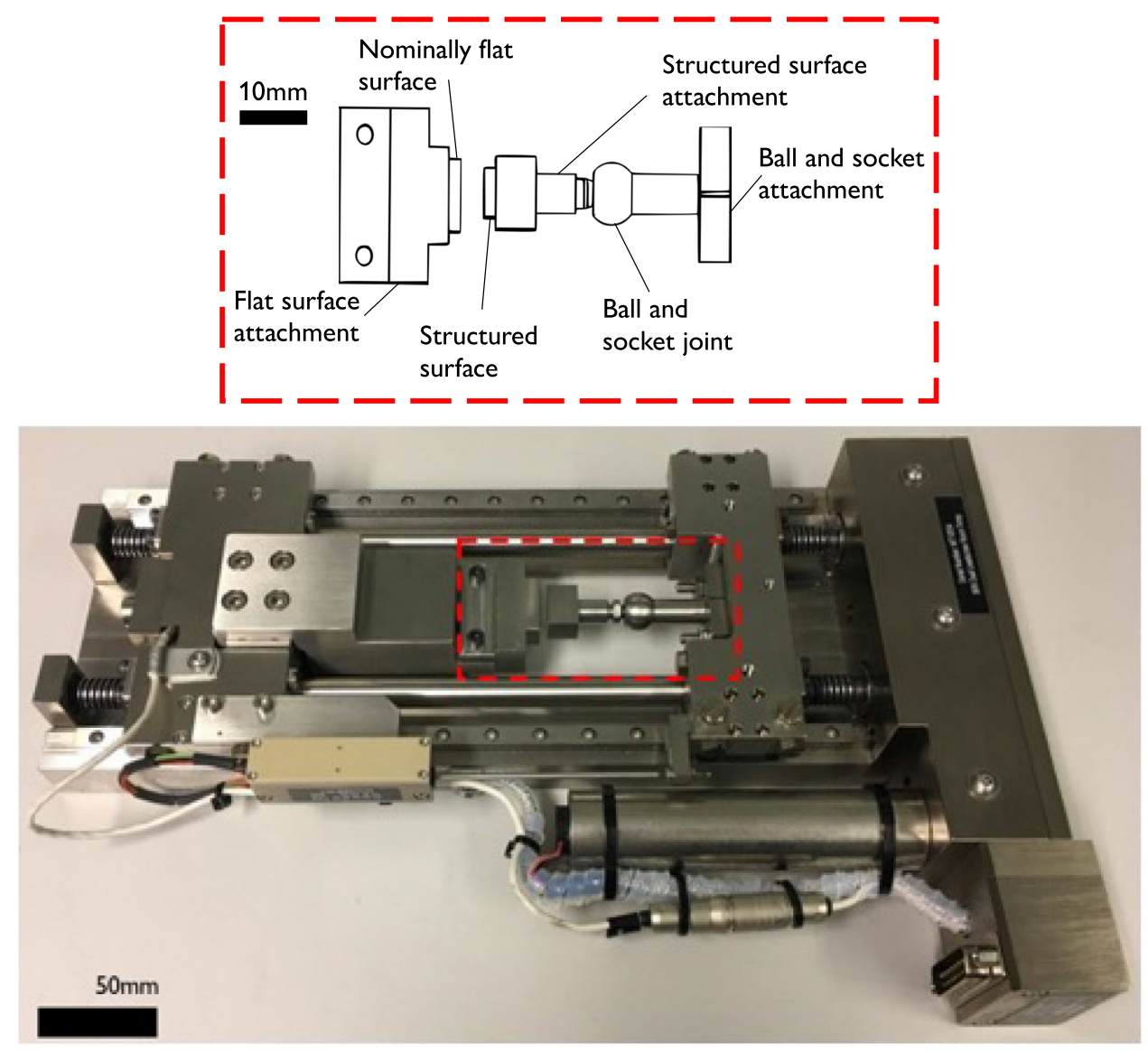

the microtester. The field of view was $6.3 \times 4.7 \mathrm{~mm}$ with $640 \times 480$ pixels giving an image resolution of $9.8 \mu \mathrm{m}$. The lens settings selected allowed for sufficient resolution while affording a large enough depth of field to keep the image in focus during the test. Imetrum Video Gauge DIC software was then used to calculate relative displacements from the videos. The software is capable of detecting displacements of 1/200th of a pixel, so the minimum resolvable displacement will be about $0.05 \mu \mathrm{m}$. The same sampling rate $(0.5 \mathrm{~s})$ was used for both load measurement and image capture allowing ease of synchronisation of load and displacement. Tests on two separate pairs of samples were each repeated five times for each of the contact area ratio cases of $\lambda_{\mathrm{f}} / \lambda_{\mathrm{p}}=0.2,0.4,0.6,0.8$ and 1 .

\section{Results and Discussion}

Although we started with 'design' values for the dimensions of the structured surface pattern, the 'as-produced' surfaces in polycarbonate differ considerably from these. Table 1 reports the changes in key surface dimensions (having a bearing on contact stiffness) from design values to as-produced polycarbonate (PC). Dimensions are given for feature depth, feature width and contact area ratio. For feature depth and width, the intermediate silicon master value is also indicated. Going from design to as-produced PC, feature depth increases by a maximum of up to $7.9 \%$ ( 0.6 design contact ratio case), whereas contact area ratio reduces by up to $20 \%$ ( 0.2 design contact ratio case). The reason for this is mostly related to elongation in feature depth and shrinkage at the tops of the features during the injection moulding stage. This is due to a combination of feature stretching during part ejection and polymer shrinkage during cooling. Note, from here on, we thus refer to two contact area ratios: design and as-produced. There will be some dimensional change in realising the intermediate master surfaces in silicon, but most of the change occurs during the polycarbonate moulding step (see Table 1). To account for this change in dimensions, the FE analysis was carried out both for the original design profile and for a modified version based on 'as-produced' dimensions. Figure 7 depicts this distinction. Figure $7 \mathrm{a}$ indicates the design waveform, and Fig. $7 \mathrm{~b}$ describes the modified waveform. The modified waveform is based on as-produced dimensions and includes linear sloped edges to approximate the non-unfirm feature shrinkage. An SEM image of a typical cleaved cross section of an as-produced PC sample is also shown in Fig. 7c for comparison. In the remainder of the paper, a distinction is made between the 
Fig. 6 Schematic representation of specimens prior to contact and zoomed-in region illustrating digital image correlation (DIC) target pairs for tracking relative displacements. The relative displacement of the interface (during the experiment) is the change in $d$ (i.e. $\Delta d$ ). The value of $d$ is initially approximately $200 \mu \mathrm{m}(100 \mu \mathrm{m}$ on each side). Five target pairs were used with only three shown here

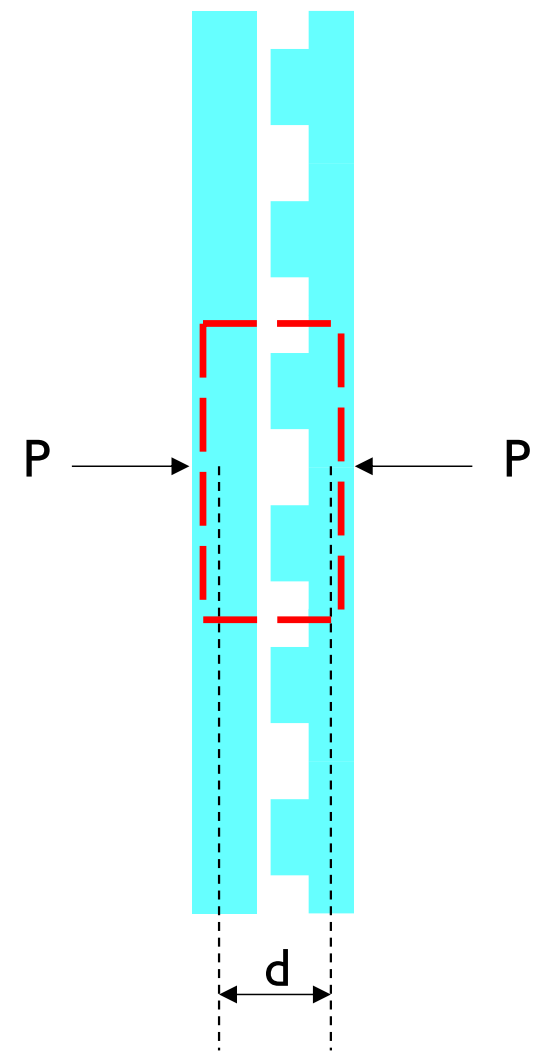

(a)

(b)

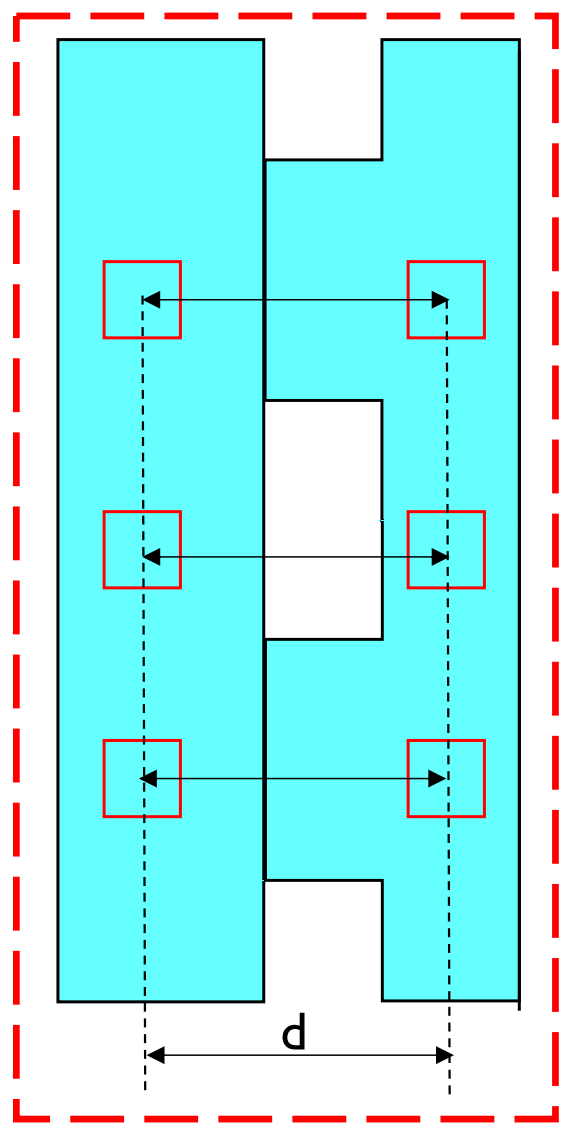

(a)

(b)

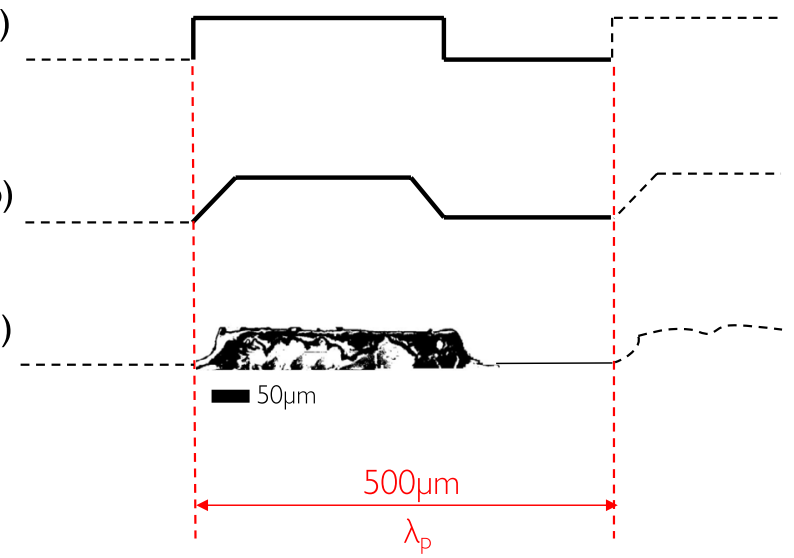

Fig. 7 (a) as-designed surface waveform based on design dimensions, (b) modified surface waveform with graduated edges based on 'as-produced' dimensions and (c) SEM image of a cleaved cross section of an 'as-produced' structured polymer sample (with scale bar included)

FE model using the 'as-designed' surface profile and the one using the approximation of the 'as-produced' surface profile. Note that, although there are some significant differences between the design and as-produced dimensions here, it would actually be straightforward to adjust and refine the fabrication process to produce dimensions far closer to the desired dimensions.

Figure 8a shows a representative plot of normal load versus relative displacement from the experiments (case shown here for design contact area ratio of 0.2). Plots for each of the five target pairs are shown together with the average trace. Note that there is some variation in the total amount of relative displacement that occurs at each target pair which may be dependent on small discrepancies in the heights of the features or with the interface alignment (or indeed on whether a target pair lines up with a square wave feature or a gap). However, the traces appear to be parallel indicating similar contact stiffness response. Figure $8 \mathrm{~b}$ gives the corresponding plot of normal contact stiffness versus normal load (as calculated from the derivative of the average trace in Fig. 8a). Note that the small-scale oscillations apparent in Fig. 8a had to be smoothed out before taking the derivative. Three zones are apparent in Fig. 8a: (i) initial large displacement due to bending to accommodate waviness and lack of flatness, (ii) load-dependent increasing interface stiffness due to flattening of surface topography (the curved part) and (iii) saturated interface stiffness (the linear part). Figure $8 \mathrm{~b}$ clearly shows the increasing contact stiffness followed by a plateau. The dashed line in Fig. $8 \mathrm{~b}$ represents the slope of the linear (saturated stiffness) region in Fig. 8a. 

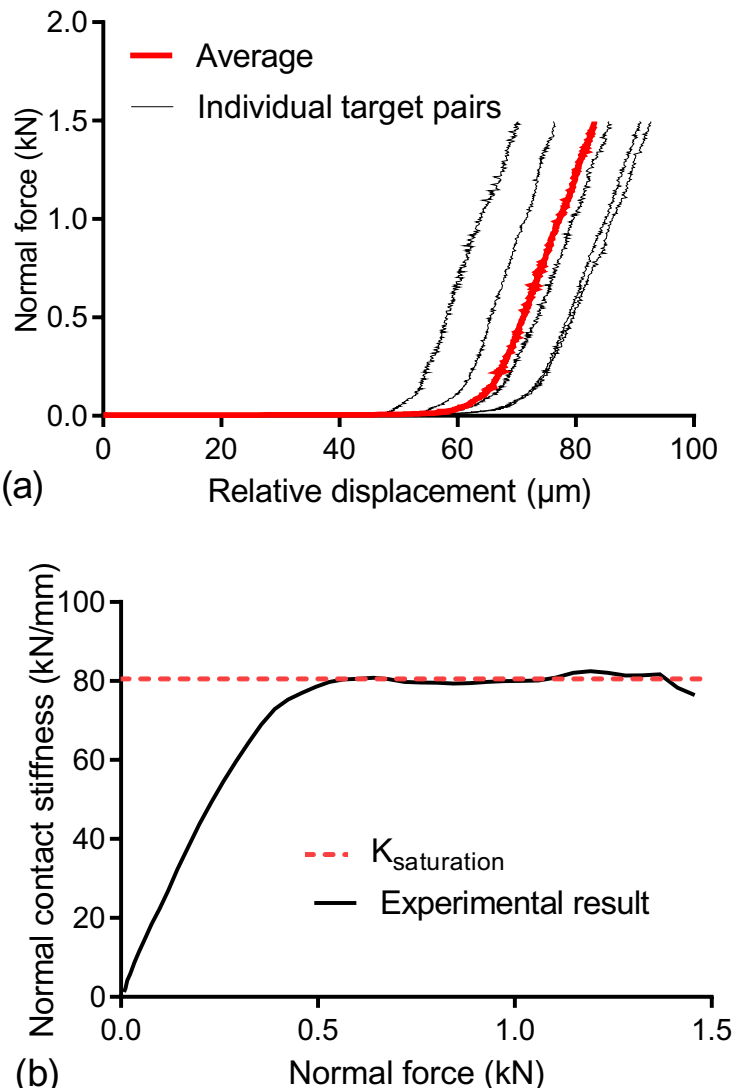

Fig. 8 a Normal force versus relative normal displacement from a typical test showing the result at each of the five target pairs as well as the average trace (contact stiffness at saturation is determined as the slope of the linear region) and $\mathbf{b}$ normal contact stiffness versus normal force for the same test as determined from the derivative of the average trace (dashed line is the slope of the linear region in a). Design contact ratio is 0.2 (i.e. as-produced contact ratio of 0.16 )

It is reasonable to assume that the saturation in the contact stiffness coincides with the effect of the surface roughness (on the tops of the features) being sufficiently flattened such that the bulk material deformation between the measured points dominates.

Figure 9 compares normal contact stiffness versus load for each of the five design contact area ratios $(0.2,0.4,0.6$, 0.8 and 1 corresponding to measured values of $0.16,0.34$, $0.55,0.77$ and 1). Despite some variation, in each case, the curves plateau to a saturated contact stiffness are corresponding to the slope of the linear region of the corresponding average trace of load versus displacement (this value is indicated by the dashed red line).

It is now reasonable to compare the saturated experimentally measured stiffness values to the stiffness values from the FE models (as the surface topography effect present on

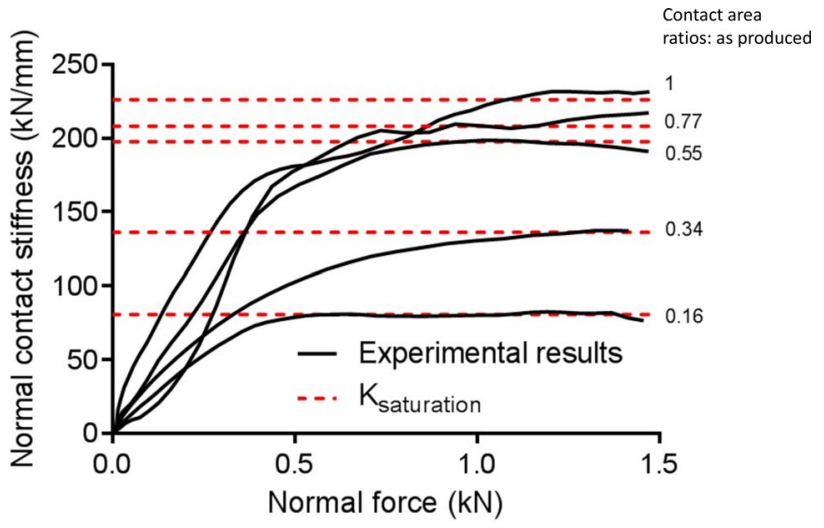

Fig. 9 Normal contact stiffness versus load for each of the five contact area ratio cases. The curves are determined from the derivative of the corresponding average force-displacement trace, and the dashed lines represent the slope of the linear part of the trace. Note the design contact area ratios $0.2,0.4,0.6,0.8$ and 1 correspond to the measured values of $0.16,0.34,0.55,0.77$ and 1 shown on the plot

the tops of the features in the experimental results should be minimised at saturation). The comparison is indicated in Fig. 10 and Table 2 where the measured results are compared with both 'as-designed' and modified 'as-produced' FE results. Note, the experimental contact stiffness results in Fig. 10 and Table 2 are the mean values of the slopes of the linear region of the load-displacement traces of the five target pairs. Owing mostly to the dimensional changes introduced during fabrication of the structured surfaces (see Table 1), the experimental contact stiffness values differ somewhat from the 'as-designed' FE values: they are 5.1-35.1\% lower according to Table 2. However, when the dimensional changes are accounted for (approximately) in

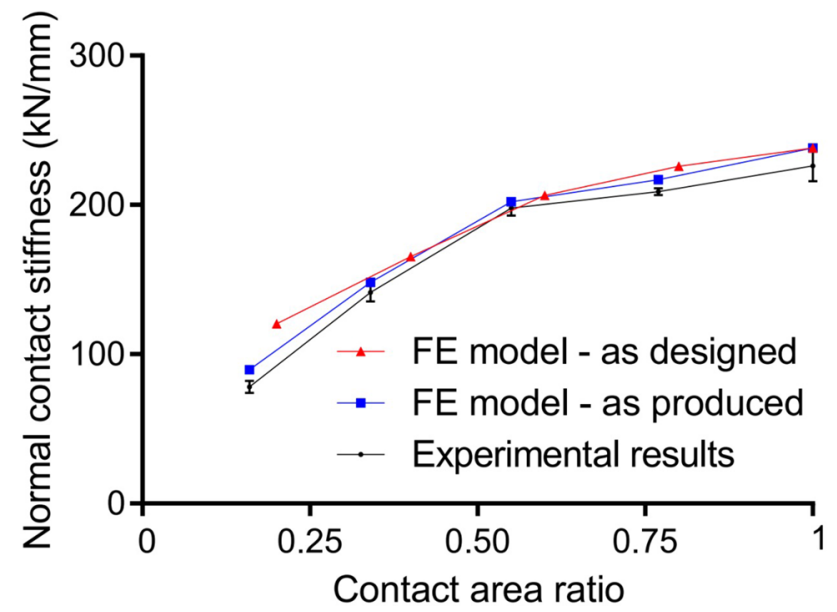

Fig. 10 Measured (total) normal contact stiffness (at saturation) versus nominal contact area ratio in comparison with 'as-designed' and modified 'as-produced' FE representations (Note contact area ratio $\left.=\frac{\lambda_{\mathrm{f}}}{\lambda_{\mathrm{p}}}\right)$ 
the FE model (the 'as-produced' case), this discrepancy reduces to a 5-12.9\% drop. Indeed, Fig. 10 shows that the experimental stiffness values closely follow the 'as-produced' FE results. In fact, the larger deviation between the measured results and the 'as-designed' case is not really a problem as the fabrication process can easily be adjusted and refined to give surface dimensions matching the design case far more closely. In that sense, the agreement of the measured results with the 'as-produced' FE results is actually a good indication of the feasibility of tailoring contact stiffness. Crucially, the measured stiffness values closely follow the FE designs in terms of both trend and magnitudes (Fig. 10). The premise is rather simple: as the contact area ratio is reduced, the width of the features is reduced, there is less material to resist the normal load, and the stiffness of the square wave interface in the normal direction is reduced. As the contact area ratio was reduced from unity to 0.2 , it was possible to reduce the contact stiffness by almost three times (Table 2). It is also very promising that the experimental results are very repeatable. Tests for each contact area ratio were repeated five times on each of two separate pairs of samples (i.e. 10 tests at each contact area ratio), and the error bars in Fig. 10 and standard deviations in Table 2 indicate excellent repeatability.

The possibility to practically fabricate interfaces with tailorable and repeatable contact stiffness (as demonstrated here for polycarbonate surfaces) has wide implications. There are several engineering scenarios where accurate control of contact stiffness is required-as mentioned, the stability of a bone-implant interface, the vibration behaviour of joints, the electrical and thermal conductance of a contact interface, the behaviour of robotic gripping systems, and the performance and control of precision machinery. The approach here can easily be expanded to fabrication of structured surfaces in other polymers via injection moulding or $3 \mathrm{D}$ printing and indeed in metals via techniques such as metal 3D printing or electroplating.

To better explain the transition of normal contact stiffness versus normal load from load dependent to a near-constant value (i.e. as in Figs. 8 and 9), the total normal contact stiffness $K$ can be decomposed as follows using the approach taken by Medina et al. [29]:

$\frac{1}{K}=\frac{1}{K_{\text {saturation }}}+\frac{1}{K_{\text {roughness }}}$,

where $K_{\text {saturation }}$ is equivalent to the bulk stiffness when the roughness is completely flattened (i.e. at sufficiently high normal load), and $K_{\text {roughness }}$ is the contribution from roughness alone. The roughness in our experiments is the roughness inevitably present on the tops of the square wave features. In the scope of linear elastic contact, the finite element model shows that $K_{\text {saturation }}$ is nearly constant regardless of the magnitude of the normal load. Therefore, $K_{\text {roughness }}$ must monotonically increase with the normal force. The monotonic increase of $K_{\text {roughness }}$ can be confirmed (for the surfaces used in the tests here) using a linear elastic boundary element method (BEM) model developed by Polonsky and Keer [30]. The rough surface topography of the polycarbonate samples was measured using an optical profilometer (Contour GT, Bruker, US) at seven different locations each with sample area of $133.5 \times 176.7 \mu^{2}$ and in-plane resolution of $0.128 \mu \mathrm{m}$. The corresponding rough surface statistics are given in Table 3. $R_{\mathrm{a}}$ and $R_{\mathrm{q}}$ are center-line average and root-mean-square roughness; $\sigma_{\mathrm{s}}$ is the standard deviation of summit heights of all asperities; and $\kappa_{\mathrm{s}}$ is the mean summit curvature of all asperities.

Assuming the mating surface (polycarbonate samples) shares the same roughness topography, the average interfacial gap and percentage of real contact area associated with a sequence of increasing normal loads (up to $700 \mathrm{~N}$ ) are calculated in Fig. 11a. The average interfacial gap here is the mean gap between two deforming (rough) contact surfaces (i.e. the rough surfaces representative of the tops of the features). The (mean) average interfacial gap gradually reduces with normal force. $K_{\text {roughness }}$ can then be determined as the absolute derivative of normal force with respect to the average interfacial gap which can be represented by the reciprocal of the slope of the average interfacial gap to normal load curve in Fig. 11a. The interfacial stiffness exponentially increases up to infinity once the roughness is completely flattened where the average interfacial gap becomes vanishingly small. However, this exponential increase is slowed down by plasticity and the multi-scale nature of roughness. Therefore, even with these deterministic structured surfaces, low levels of surface roughness present on the tops of the features $\left(R_{\mathrm{a}} \approx 0.026 \mu \mathrm{m}\right)$ are responsible for the load-dependent contact stiffness in the lower load regime. This means that the tailorable aspect discussed above is mostly only applicable above the load required to produce a load independent saturated contact stiffness. Now, using Eq. 1, the total normal contact stiffness $K$ predicted by the BEM can now be obtained using the $K_{\text {roughness }}$ predicted by the BEM (at each load) and using $K_{\text {saturation }}=80.5 \mathrm{kN} / \mathrm{mm}$ (from the experiments). Compared with the experimental results shown in Fig. 11b, the BEM prediction shows a similar convergent trend as normal force increases, but with a much steeper slope. This is a strong signal that $K_{\text {roughness }}$ is overestimated

Table 3 Statistics of the measured rough surface: $R_{\mathrm{a}}$ and $R_{\mathrm{q}}$ are center-line average and root-mean-square roughness, $\sigma_{\mathrm{s}}$ is the standard deviation of summit heights of all asperities, and $\kappa_{\mathrm{s}}$ is the mean summit curvature of all asperities

\begin{tabular}{llll}
\hline$R_{\mathrm{a}}(\mu \mathrm{m})$ & $R_{\mathrm{q}}(\mu \mathrm{m})$ & $\sigma_{\mathrm{s}}(\mu \mathrm{m})$ & $\kappa_{\mathrm{s}}(1 / \mu \mathrm{m})$ \\
\hline $0.026 \pm 0.007$ & $0.048 \pm 0.008$ & $0.040 \pm 0.004$ & $0.071 \pm 0.009$ \\
\hline
\end{tabular}


(a)

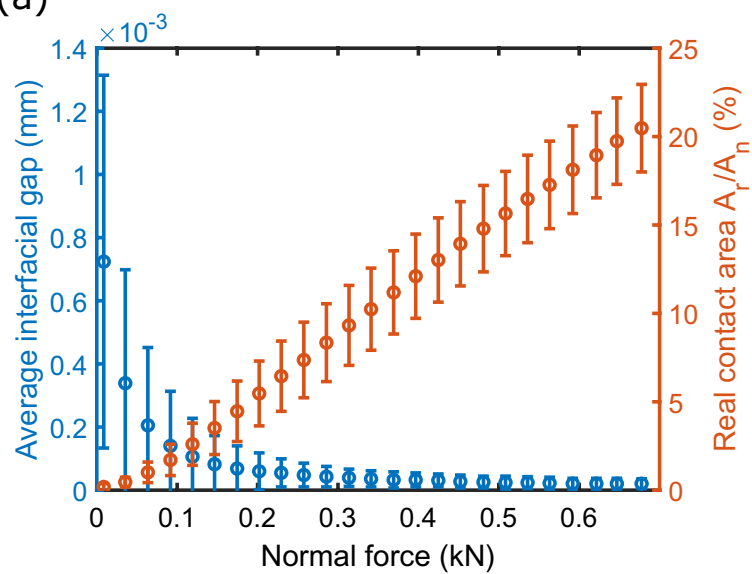

Fig. 11 a Evolution of average interfacial gap (left $y$-axis) and percentage of real contact area (right $y$-axis) with respect to the normal force for the BEM model of the rough surface polycarbonate contact pair and $\mathbf{b}$ comparison of the measured and numerical (BEM) total

by the BEM. The major reason for this is probably the neglection of the plastic deformation at asperity level. The plastic deformation of the rough (isotropic) surface can be quantified using the plasticity index [18]:

$\psi=\frac{E^{*}}{H} \sqrt{\sigma_{\mathrm{s}} \kappa_{\mathrm{s}}}$,

where $E^{*}=\frac{E_{\mathrm{PC}}}{2\left(1-v_{\mathrm{PC}}^{2}\right)}$ is the effective modulus of PC contact pair; $H$ is the hardness of $\mathrm{PC}$ which can be estimated using $H=2.8 S_{\mathrm{y}}$ where $S_{\mathrm{y}}$ is the yield strength of PC. Using $E_{\mathrm{PC}}=2350 \mathrm{MPa}, S_{\mathrm{y}}=60 \mathrm{MPa}$ and values of $\sigma_{\mathrm{s}}$ and $\kappa_{\mathrm{s}}$ in Table 3 , we get the plasticity index $\psi=1.41>1$ which indicates that the asperity deformation is predominantly plastic. According to Fig. 11b, the experimental normal contact stiffness starts to converge at the normal force of $400 \mathrm{~N}$ where the real contact area is predicted by the BEM to only occupy approximately $13 \%$ (Fig. 11a) of the entire nominal contact area. If plastic deformation is included, a much higher percentage of real contact area may be achieved, as well as a lower interfacial gap. This would have the effect of decreasing the interfacial stiffness and softening the total normal BEM contact stiffness curve towards the experimental curve.

\section{Conclusions}

A novel practical approach to realising interfaces with tailored and repeatable normal contact stiffness using micropatterned surfaces has been developed. The paper uses a finite element model to first design square wave interfaces having a range of controllable contact stiffnesses and then fabricates these in polycarbonate via a microfabrication process (b)

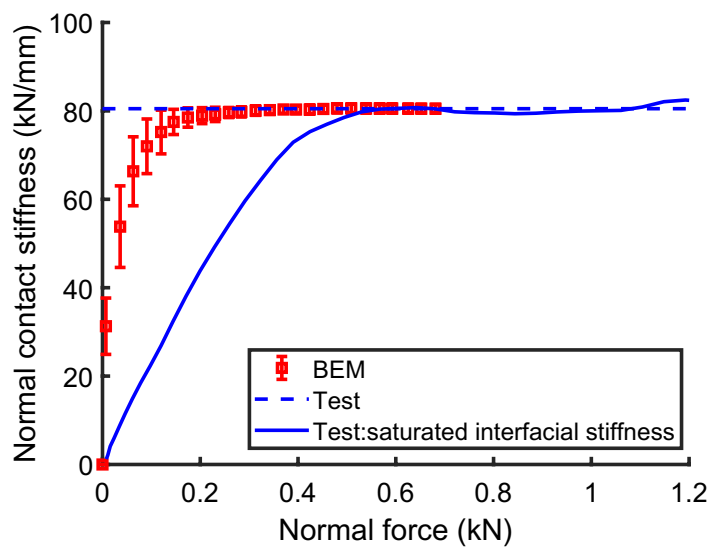

normal contact stiffness results. Note the average interfacial gap here is the mean gap between two deforming (rough) contact surfaces (i.e. the rough surfaces representative of the tops of the features)

involving steps such as photolithography, etching, microimprinting and injection moulding. The 'as-produced' surface dimensions, and consequently the measured contact stiffnesses, differed somewhat from the original starting design values, but this is not a problem because the fabrication process can easily be refined to produce surface profiles having the desired dimensions. In this case, rather than adjusting the process, we adjusted the FE model to accommodate changes introduced during fabrication. Results indicate that the experimental contact stiffness values closely follow the FE predictions in terms of magnitudes and trend. The results were also highly repeatable both between new test samples and between repeat tests. The idea is simple: by adjusting the widths of the features, the contact stiffness can be controlled. In the present work, it was possible to reduce the contact stiffness by almost three times going from a contact area ratio of unity to 0.2 . This capability to produce interfaces with tailorable and repeatable contact stiffness will be useful in several engineering applications where contact stiffness is important: bone-implant interfaces, vibration of mechanical joints, operation of robotic gripping systems and the performance and control of precision devices. The approach presented here can be expanded to other polymers via injection moulding and 3D printing and to metals via approaches such as metal 3D printing.

Even with these deterministic microstructured surfaces, surface roughness present on the tops of the features $\left(R_{\mathrm{a}} \approx 0.03 \mu \mathrm{m}\right)$ was shown to produce a load-dependent contact stiffness in the lower load regime. In the tests in the present work, the higher loads applied were sufficient to flatten the roughness and produce a load independent saturated contact stiffness. Therefore, the tailorable aspect is mostly applicable beyond a certain load limit when the stiffness 
reaches its plateau value. Comparison of the experimental results with elastic predictions made by applying the boundary element method to the measured roughness shows that the elastic prediction produces a significantly more rapid increase in total contact stiffness with load than the experimental measurements. Therefore, it is suggested that plastic deformation of the rough surface topography in the experimental case is responsible for the more compliant measured interface response.

Acknowledgements The authors would like to acknowledge the support of the Leverhulme Trust for funding the work via Project Grant "Fundamental Mechanical Behaviour of Nano and Micro Structured Interfaces" (RPG-2017-353). The Leverhulme Trust is also acknowledged for providing the $\mathrm{PhD}$ studentship for the first author. We would also like to thank the technical staff at the James Watt Nanofabrication Centre (JWNC) at the University of Glasgow for assistance in fabricating the samples. Mr Alex Hamilton is thanked for useful discussions throughout the work.

Funding This study was funded by Leverhulme Trust Project Grant No. RPG-2017-353.

Data Availability Data will be made available via an online university data repository.

Code Availability Not applicable.

\section{Declarations}

Conflict of interest The authors declare that they have no conflict of interest.

Open Access This article is licensed under a Creative Commons Attribution 4.0 International License, which permits use, sharing, adaptation, distribution and reproduction in any medium or format, as long as you give appropriate credit to the original author(s) and the source, provide a link to the Creative Commons licence, and indicate if changes were made. The images or other third party material in this article are included in the article's Creative Commons licence, unless indicated otherwise in a credit line to the material. If material is not included in the article's Creative Commons licence and your intended use is not permitted by statutory regulation or exceeds the permitted use, you will need to obtain permission directly from the copyright holder. To view a copy of this licence, visit http://creativecommons.org/licenses/by/4.0/.

\section{References}

1. Raffa, M.L., Nguyen, V.-H., Haiat, G.: Micromechanical modeling of the contact stiffness of an osseointegrated bone-implant interface. Biomed. Eng. Online 18(1), 114 (2019)

2. Brake, M.R.W.: The Mechanics of Jointed Structures: Recent Research and Open Challenges for Developing Predictive Models for Structural Dynamics. Springer, Cham (2018)

3. Sevostianov, I., Kachanov, M.: Contact of rough surfaces: a simple model for elasticity, conductivity and cross-property connections. J. Mech. Phys. Solids 56(4), 1380-1400 (2008)
4. Barber, J.R.: Bounds on the electrical resistance between contacting elastic rough bodies. Proc. R. Soc. Lond. Ser. A Math. Phys. Eng. Sci. 459(2029), 53-66 (2003)

5. Cutkosky, M.R., Wright, P.K.: Friction, stability and the design of robotic fingers. Int. J. Robot. Res. 5(4), 20-37 (1986)

6. Carbone, G.: Grasping in Robotics. Springer, London (2013)

7. Zou, H.T., Wang, B.L.: Investigation of the contact stiffness variation of linear rolling guides due to the effects of friction and wear during operation. Tribol. Int. 92, 472-484 (2015)

8. Kendall, K., Tabor, D.: An ultrasonic study of the area of contact between stationary and sliding surfaces. Proc. R. Soc. Lond. Ser. A Math. Phys. Sci. 323(1554), 321-340 (1971)

9. Drinkwater, B.W., Dwyer-Joyce, R.S., Cawley, P.: A study of the interaction between ultrasound and a partially contacting solid-solid interface. Proc. Math. Phys. Eng. Sci. 452(1955), 2613-2628 (1996)

10. Dwyer-Joyce, R.S., Drinkwater, B.W., Quinn, A.M.: The use of ultrasound in the investigation of rough surface interfaces. J. Tribol. 123(1), 8-16 (2001)

11. Filippi, S., Akay, A., Gola, M.: Measurement of tangential contact hysteresis during microslip. J. Tribol. 126, 482-489 (2004)

12. Serpe, C.I.: The role of contact compliance in the deformation, wear and elastic stability of metallic sliding rings: experiments and computational analysis. Ph.D. Dissertation, State University of New York at Buffalo, NY (1999)

13. Kartal, M.E., Mulvihill, D.M., Nowell, D., Hills, D.A.: Determination of the frictional properties of titanium and nickel alloys using the digital image correlation method. Exp. Mech. 51(3), 359-371 (2011)

14. Kartal, M.E., Mulvihill, D.M., Nowell, D., Hills, D.A.: Measurements of pressure and area dependent tangential contact stiffness between rough surfaces using digital image correlation. Tribol. Int. 44(10), 1188-1198 (2011)

15. Mulvihill, D.M., Brunskill, H., Kartal, M.E., Dwyer-Joyce, R.S., Nowell, D.: A comparison of contact stiffness measurements obtained by the digital image correlation and ultrasound techniques. Exp. Mech. 53(7), 1245-1263 (2013)

16. Polycarpou, A.A., Etsion, I.: Analytical approximations in modeling contacting rough surfaces. J. Tribol. 121(2), 234-239 (1999)

17. Shi, X., Polycarpou, A.A.: Measurement and modeling of normal contact stiffness and contact damping at the meso scale. J. Vib. Acoust. 127(1), 52-60 (2005)

18. Greenwood, J.A., Williamson, J.B.P.: Contact of nominally flat surfaces. Proc. R. Soc. Lond. Ser. A Math. Phys. Sci. 295(1442), 300-319 (1966)

19. Persson, B.N.J.: Relation between interfacial separation and load: a general theory of contact mechanics. Phys. Rev. Lett. 99(12), 125502 (2007)

20. Pei, L., Hyun, S., Molinari, J.F., Robbins, M.O.: Finite element modeling of elasto-plastic contact between rough surfaces. J. Mech. Phys. Solids 53(11), 2385-2409 (2005)

21. Pohrt, R., Popov, V.L.: Normal contact stiffness of elastic solids with fractal rough surfaces. Phys. Rev. Lett. 108(10), 104301 (2012)

22. Pastewka, L., Prodanov, N., Lorenz, B., Müser, M.H., Robbins, M.O., Persson, B.N.J.: Finite-size scaling in the interfacial stiffness of rough elastic contacts. Phys. Rev. E 87(6), 062809 (2013)

23. Sever, I.A.: Experimental validation of turbomachinery blade vibration predictions. Ph.D. Dissertation, Imperial College London (2004)

24. Barber, J.R.: Incremental stiffness and electrical contact conductance in the contact of rough finite bodies. Phys. Rev. E 87(1), 013203 (2013) 
25. Jaber, S.B., Hamilton, A., Xu, Y., Kartal, M.E., Gadegaard, N., Mulvihill, D.M.: Friction of flat and micropatterned interfaces with nanoscale roughness. Tribol. Int. 153, 106563 (2021)

26. Hamilton, A., Xu, Y., Kartal, M.E., Gadegaard, N., Mulvihill, D.M.: Enhancing strength and toughness of adhesive joints via micro-structured mechanical interlocking. Int. J. Adhes. Adhes. 105, 102775 (2021)

27. Li, S., Yao, Q., Li, Q., Feng, X.-Q., Gao, H.: Contact stiffness of regularly patterned multi-asperity interfaces. J. Mech. Phys. Solids 111, 277-289 (2018)

28. Hamilton, A., Perris, J., Convery, N., Mulvihill, D.M., Gadegaard, N.: Flexible inserts for injection molding of complex micro-structured polymer components. Macromol. Mater. Eng. (2021) (In Press)

\section{Authors and Affiliations}

\section{Jack Perris $^{1} \cdot$ Yang X $\mathbf{u}^{2} \cdot$ Mehmet E. Kartal $^{3} \cdot$ Nikolaj Gadegaard $^{1}$ - Daniel M. Mulvihill ${ }^{1}$ (b)}

1 James Watt School of Engineering, University of Glasgow, Glasgow G12 8QQ, UK

3 School of Engineering, University of Aberdeen, Aberdeen AB24 3UE, UK

2 School of Mechanical Engineering, Hefei University of Technology, Hefei 230009, China

29. Medina, S., Nowell, D., Dini, D.: Analytical and numerical models for tangential stiffness of rough elastic contacts. Tribol. Lett. 49(1), 103-115 (2013)

30. Polonsky, I.A., Keer, L.M.: A numerical method for solving rough contact problems based on the multi-level multi-summation and conjugate gradient techniques. Wear 231(2), 206-219 (1999)

Publisher's Note Springer Nature remains neutral with regard to jurisdictional claims in published maps and institutional affiliations. 